\title{
Lacrimal Gland Intra-Lobular Duct Cysts Associated with Focal Vasculitis
}

\author{
Hardeep Singh Mudhara Zanna I. Currie ${ }^{b}$ Sachin M. Salvi ${ }^{b}$ \\ a National Specialist Ophthalmic Pathology Service (NSOPS), Department of Histopathology, \\ and ${ }^{\mathrm{b}}$ Department of Ophthalmology, Royal Hallamshire Hospital, Sheffield, UK
}

\section{Key Words}

Lacrimal gland · Vasculitis · Lacrimal ductule cyst

\begin{abstract}
Purpose: Description of the clinical and histopathological features of unusual lacrimal gland intra-glandular duct cysts. Procedures: A 38-year-old male presented with bilateral upper lid lumpiness, which was worse on the left. Computed tomography scan showed bilateral multiple lacrimal gland cysts, which were larger on the left compared to the right. After two unsuccessful attempts to excise the largest cyst on the left side, it was removed at the third attempt using a novel technique that incorporated the use of fibrin glue to fill the remaining cavity. Results: The microscopy of the left-sided cyst comprised a cavity containing fibrin glue, lined by intra-lobular lacrimal gland duct epithelium. The cyst wall contained reactive lymphoid aggregates, plasma cells and eosinophils associated with fibrosis. Focally, there were small vessels affected by an acute vasculitis associated with eosinophils and a granulomatous component. Conclusions: We ascribe the cyst formation to the effects of tractional fibrosis secondary to focal vasculitis and to obstructive fibrosis of the lacrimal ductules. This case also described a novel use of Tisseel fibrin glue to assist intact removal of a lacrimal gland cyst.

(C) 2015 S. Karger AG, Basel
\end{abstract}

\section{Introduction}

Many types of orbital cysts have been described. These include ductular cysts of the lacrimal gland, microphthalmos with cyst, epidermoid and dermoid cysts, encephaloceles and mucocoels, cystic vascular and lymphatic lesions, haematic cysts, aneurysmal bone cysts, as well as infectious (cysticercosis and echinococcosis) and malignant tumours with cystic changes [1]. The commonest cyst of the lacrimal gland is dacryops (cysts of the palpebral lacrimal gland ductules) [2], which is often associated with previous inflammation. Bullock et 
al. [2] have classified lacrimal gland duct cysts into four types: type 1 palpebral lobe cysts; type 2 orbital lobe cysts; type 3 accessory lacrimal gland cysts, and type 4 ectopic lacrimal gland cysts. We describe the unique lacrimal gland pathology of lacrimal gland intra-lobular duct cysts associated with vasculitis.

\section{Case Report}

A 38-year-old male presented with lumps in the lacrimal gland region of both sides, which had started to appear at the age of 16 years and were associated with aching. They increased in size, progressively but slowly over the ensuing years. Recently, he has noticed a significant increase in the larger lesion on the left side, which caused discomfort and impairment of his left peripheral visual field (fig. 1a, b).

His past medical history included asthma, controlled with inhalers, and hepatitis A due to intra-venous recreational drug use in his early 20s. A computed tomography (CT) scan showed bilateral multiple lacrimal gland cysts, which were larger on the left compared to the right and affecting the orbital and palpebral parts of the glands (fig. 1c, d). There were two excision attempts of the largest left-sided cyst, initially via a skin crease incision and subsequently with a trans-conjunctival approach. However, it was difficult to excise it intact and there was subsequent recurrence. At the third attempt, the cyst was removed intact using a novel surgical method. After a lateral orbitotomy, Tisseel fibrin glue was injected into the remaining cyst cavity to fill it out, facilitating intact cyst removal.

Tissue obtained by the first trans-conjunctival approach showed serpiginous-shaped cysts (fig. 1e), lined by lacrimal gland intra-lobular duct bilayered epithelium (fig. 1f). The wall of the cysts comprised layered collagen tissue. The soft tissue around the cysts contained fragments of Müller's muscle and lacrimal gland lobules (fig. 1g).

The tissue obtained from the successful removal of the large left-sided cyst showed a striking histological appearance. Centrally, there was a cyst, containing layered eosinophilic material representing the fibrin glue (fig. $1 \mathrm{~h}$, large arrow) lined by an attenuated layer of epithelium, identical in nature to the bilayered epithelium lining the intra-lobular lacrimal gland ducts (fig. 1i). The cyst wall showed fibrosis and numerous nodular lymphoid aggregates (fig. 1j), variably atrophic but preserved lacrimal gland lobules and intralobular ductless (fig. 1k, l). Unexpectedly, in one part of the fibrous cyst wall, there was an unequivocal focal small vessel active vasculitis affecting numerous small vessel profiles (fig. $1 \mathrm{~m}$ ) featuring eosinophils and a granulomatous component (fig. 1n). Elsewhere focal fibrous obliteration of small vessels was seen as indicative of scarring from previous vasculitis episodes. Fibrosis was also narrowing inter-lobular lacrimal ductules. No infectious agent was identified. The surrounding fibrous tissue contained prominent numbers of eosinophils and plasma cells (fig. 10, p). Immunohistochemical staining with IgG4 showed no significant plasma cell positivity.

In light of the microscopic findings, a thorough autoimmune and vasculitis workup was suggested. However, the patient failed to appear for the blood tests and was lost to follow-up.

\section{Discussion}

Bullock et al. [2] classified lacrimal duct cysts into four categories: category 1 represents simple dacryops and is the commonest situation. Cyst formation is often preceded by inflammation or trauma to the site. Histology can feature chronic inflammation around the cyst with or without fibrosis. Category 2 is very rare and the most serious clinically causing globe displacement and orbital wall expansion. It is often congenital but can present in later life in adults and its pathogenesis is uncertain. Bullock et al. described the histology of one case as a thin-walled cyst lined by flattened to cuboidal epithelium thrown into papillae, adjacent to lacrimal ducts. Category 3 comprises cysts of the glands of Krause and Wolfring and occurs in the fornices. Category 4 cysts are choristomatous cysts that can be located in the conjunctiva, the orbit as well as in an intra-ocular location and which can be associated with chronic inflammatory changes. 
Ocular Oncology and Pathology

\begin{tabular}{l|l}
\hline Ocul Oncol Pathol 2015;1:225-230 \\
\hline DOI: 10.1159/000375255 & $\begin{array}{l}\text { @ 2015 S. Karger AG, Basel } \\
\text { www.karger.com/oop }\end{array}$ \\
\hline
\end{tabular}

Singh Mudhar et al.: Lacrimal Gland Intra-Lobular Duct Cysts Associated with Focal Vasculitis
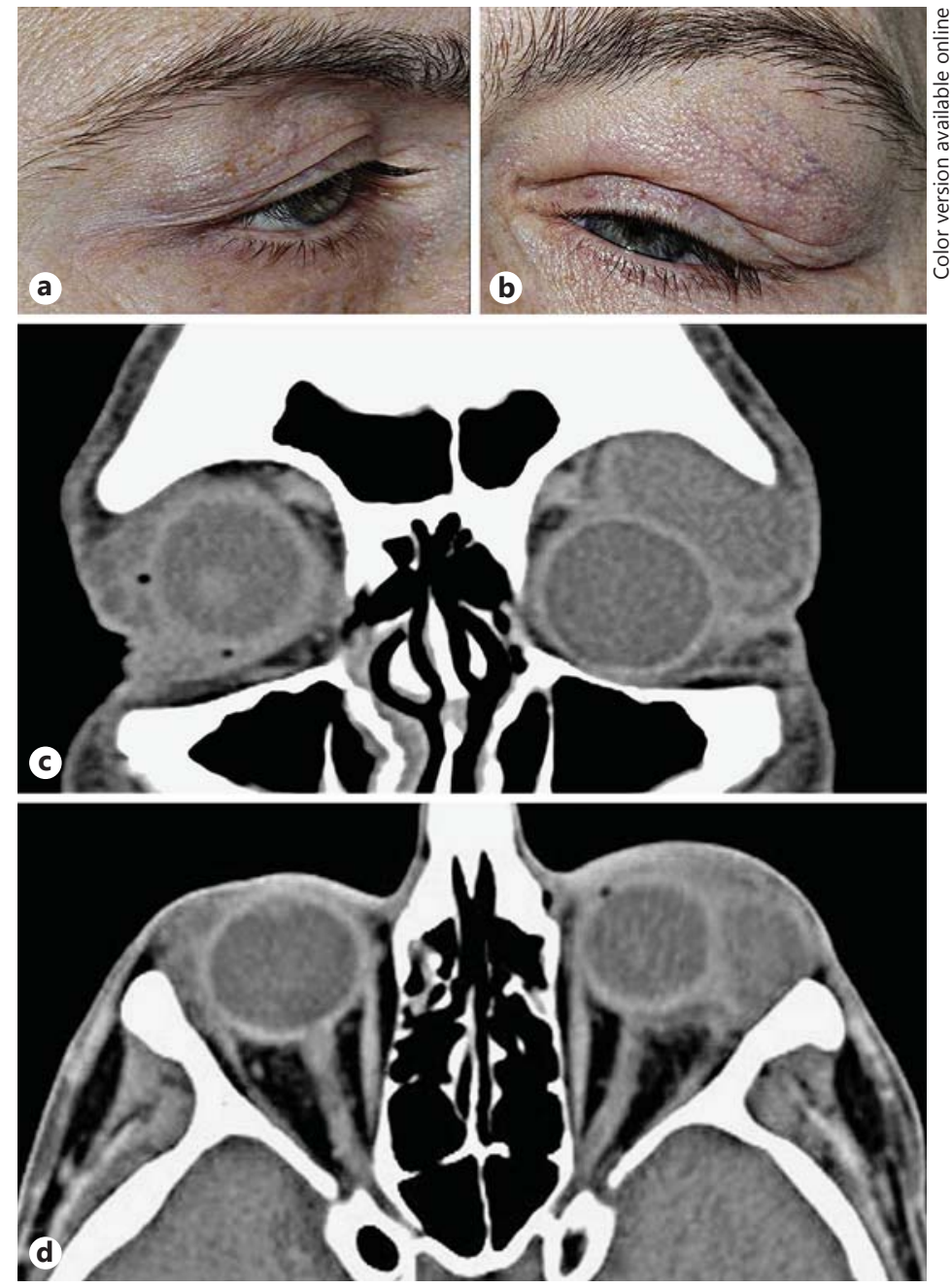

Fig. 1. a Photograph demonstrating the cyst involving the palpebral portion of the right lacrimal gland protruding through the upper lid. b Photograph demonstrating the much larger cyst involving the palpebral portion of the left lacrimal gland, seen as a diffuse fullness of the upper lid. Note the lateral ptosis and inferomedial displacement of the left globe. c Coronal section of the pre-operative CT scan demonstrating the bilateral lacrimal gland cysts. The left-sided cyst is noted to be occupying the anterior orbit without bony erosion but causing infero-medial globe displacement. $\mathbf{d}$ Axial section of a pre-operative CT scan demonstrating the bilateral lacrimal gland cysts in the palpebral and orbital part with the left cyst causing infero-medial globe displacement. e Haematoxylin and eosin (HE)-stained tissue of specimen from the previous attempt to remove the largest left-sided cyst. Small, serpiginous cysts are shown at lower power. $\mathbf{f}$ HE. Higher power of e showing the serpiginous cysts lined by bilayered epithelium of intra-lobular lacrimal gland ductules. $\mathbf{g}$ HE. Lacrimal gland tissue is present in the wall of the serpiginous cysts. $\mathbf{h}$ HE. Low power of the specimen from the successful third attempt to remove the largest left lacrimal gland cyst. The cyst lumen contains eosinophilic Tisseel fibrin glue (large arrow). The wall of the cyst is composed of eosinophilic fibrous tissue (thin arrow) and is peppered with lymphoid aggregates (small far arrow). $\mathbf{i}$ HE. The cyst in $\mathbf{h}$ is lined by ductuletype attenuated bilayered epithelium. $\mathbf{j}$ HE. This image shows the whorled collagenous fibrotic tissue containing reactive lymphoid aggregates. $\mathbf{k}$ HE. The wall of the cyst contains atrophic lacrimal gland elements. I HE. The lacrimal gland elements within the wall of the cyst are shown at higher power. Note the intra-lobular ductules (arrows). $\mathbf{m}$ HE. This image shows focal small vessel active vasculitis. The vessel wall and lumen are obliterated by neutrophils, eosinophils and macrophages. The arrows point to the original outline of the vessel. n HE. A granuloma is shown. Note the 'slipper-shaped' nuclei of epithelioid histiocytes. o HE. The cyst wall contains a prominent number of eosinophils. $\mathbf{p}$ HE. The cyst wall contains a prominent number of plasma cells.

(For figure 1e-p see next page.) 
Ocular Oncology

and Pathology
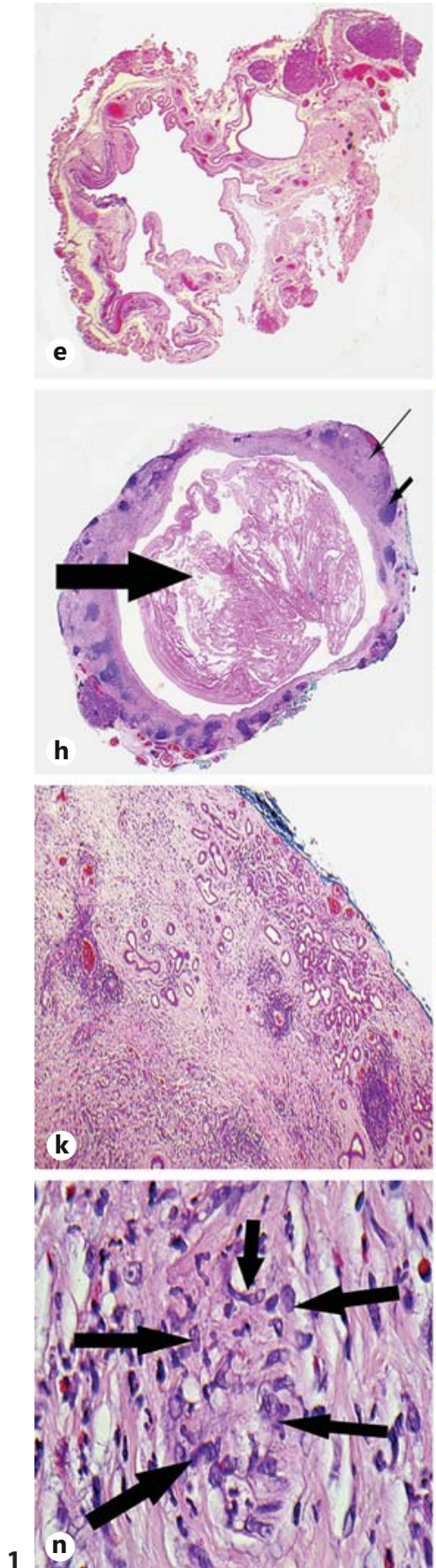

Ocul Oncol Pathol 2015;1:225-230

Singh Mudhar et al.: Lacrimal Gland Intra-Lobular Duct Cysts Associated with Focal Vasculitis
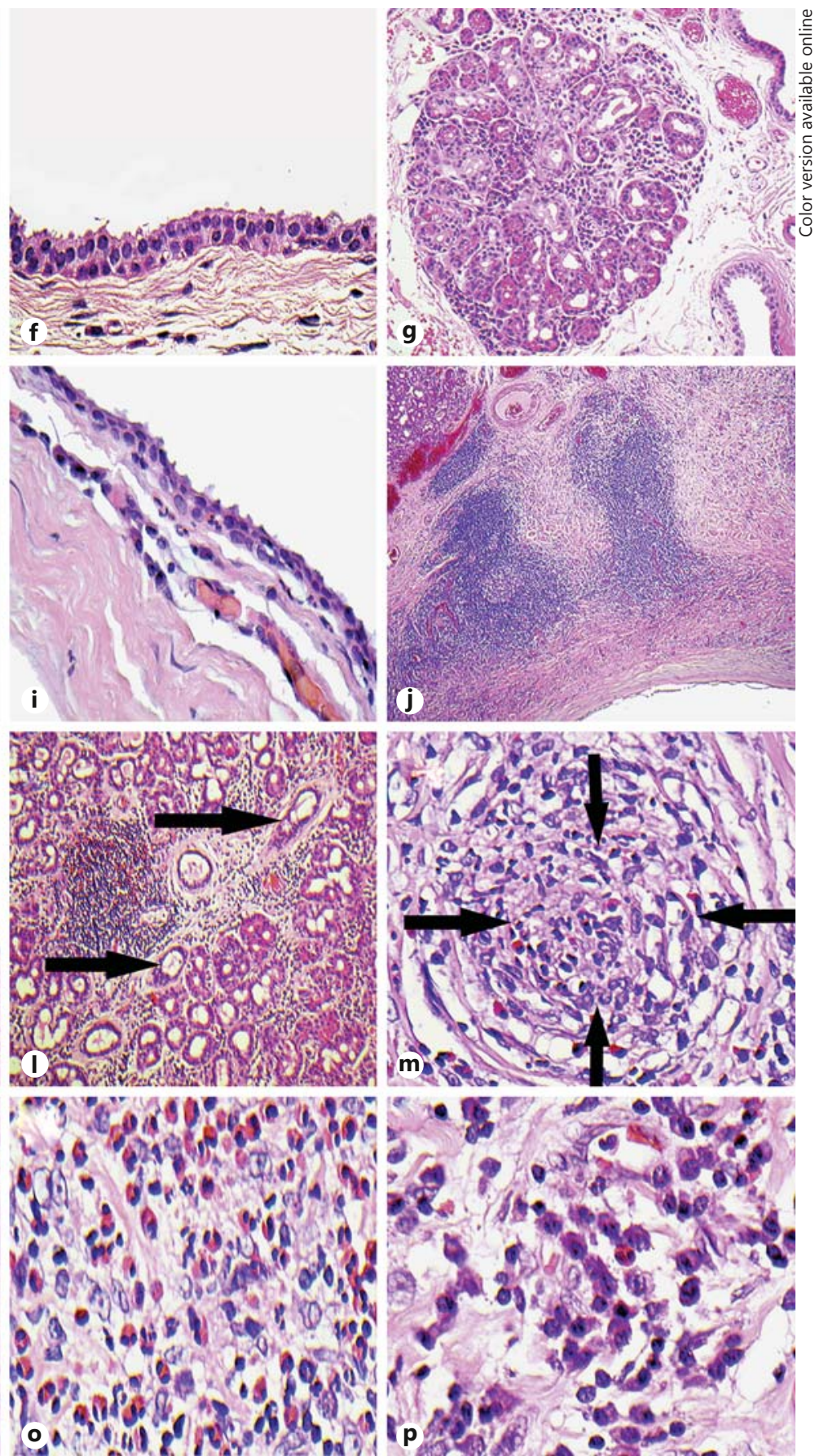

In our patient, the cysts clinically occurred in the palpebral and orbital portion of the lacrimal gland and were clearly derived from the intra-lobular lacrimal ducts. The presence of lacrimal gland elements in the wall of the cysts confirmed their lacrimal gland origin. This effectively excluded cysts such as dermoids, epidermoids, mucocoels and conjunctiva-derived cysts. The largest cyst was supported by a band of established fibrous tissue and beyond this, the wall contained lacrimal gland and inflammatory elements, with focal acute vasculitis. We suggest that the intra-lobular duct-derived cyst on the left side developed over a considerable period of time, secondary to the tractional effects of the surrounding lacrimal gland fibrosis 
as well as secondary to focal small vessel vasculitis and with a contribution from the obstructive effects of the fibrosis on extra-lobular ductules causing upstream dilation of the intra-lobular ductules. The pathology described is closest in appearance to Bullock's category 2 but with some unique features as described. While we can be reasonably certain about the pathology on the left side, the precise nature of the pathology present in the right gland cannot be histologically confirmed because it was not biopsied, although radiologically a similar process appears to be present in the right gland too.

Focal vasculitis affected small/capillary sized vessels and fits with either polyangitis with granulomatosis or with Churg-Strauss vasculitis, both of which can be granulomatous histologically and can feature eosinophils. Unfortunately, the patient did not attend the clinic to undergo serological testing for confirmatory auto-antibody profiling. The history of asthma is interesting, which classically occurs in the context of Churg-Strauss vasculitis. However, the patient also had a history of hepatitis A, which can also be associated with small vessel vasculitis of a pattern seen in this case. IgG4 can be linked with polyangitis with granulomatosis type vasculitis, but the number of IgG4 plasma cells was not significantly raised in the examined tissues.

A final possibility is that the previous two surgery attempts may have triggered a focal small vessel vasculitis with an eosinophilic and granulomatous inflammatory response. However, careful scrutiny of the histopathology showed scarring that was not effacing the existing lacrimal gland glandular and ductular elements, whereas post-surgical scarring would be expected to cause effacement of the normal anatomy. In addition, a period of around 12 months had elapsed after the second surgery, making it is unlikely that this was the cause of the acute focal vasculitis and tissue inflammatory response, as any post-surgical tissue effects should have subsided after this period of time.

There are two key histological differential diagnoses that merit further discussion. The first is sclerosing polycystic adenosis [3] which has very recently been described in the lacrimal gland and lymphoepithalial cysts, and which is especially pertinent given this patient's history of intra-venous drug usage and potential HIV infection. Sclerosing polycystic adenosis occurs classically in major and minor salivary glands and resembles fibrocystic disease of the breast. It comprises hyperplasia of ductal and acinar elements with apocrine metaplasia, cystic ducts and sclerosis. The CT scan of the case reported by Pfeiffer et al. [3] showed multiple cysts in the right lacrimal gland, not too dissimilar to the CT scan of the right lacrimal gland in our case. However, there was no evidence of confluent ductule and acinar hyperplasia and no apocrine metaplasia.

HIV-associated cystic lymphoid hyperplasia shows dense lymphoid tissue (that can easily be mistaken for low-grade mucosa-associated lymphoid tissue lymphoma) abutting the epithelial lining of the cyst, which invariably is stratified and squamous in type and classically affects the parotid glands, although no cases have been described in the lacrimal gland [4]. In our case, the cyst was supported by a distinct band of fibrosis, and the lymphoid tissue did not border the epithelial lining. Besides, the lymphoid tissue was not dense enough to be considered as a serious contender.

Small palpebral lacrimal gland cysts are normally observed even if they are not causing any problems as they have a high risk of recurrence. In our patient, the large left-sided lacrimal gland cyst had started to cause discomfort, ache, pressure effects on the globe causing distortion of vision and impairment of the peripheral field of vision, necessitating surgical excision. The first approach was via an upper lid skin crease incision, but the cyst ruptured towards the end of surgery and recurred. The second time, a trans-conjunctival approach was attempted but again it ruptured leading to recurrence. At the third attempt, a novel technique was utilised. Tisseel fibrin glue has been documented to be useful in removing lymphangiomas which often display thin, fragile walls [5]. Utilising this approach and knowing that 
the cyst had ruptured on two previous occasions, the left-sided large lacrimal gland cyst was partially drained and then made firm with the injection of Tisseel fibrin glue and excised completely via a lateral orbitotomy approach. On follow-up, there was no evidence of cyst recurrence and the patient's symptoms had improved. However, the patient did not attend the follow-up appointments, presumably as he was satisfied with the result of his last surgery.

In summary, we have described the clinical and histopathological features of a novel lacrimal gland cystic lesion, associated with focal small vessel vasculitis. This unique pathology was only made possible to assess because of the adoption of a novel surgical approach which employed the use of Tisseel fibrin glue to fill the cyst space allowing an intact excision.

\section{Disclosure Statement}

The authors have no conflicts of interest to disclose.

\section{References}

1 Borodic GE: Cystic lesions of the orbit; in Albert DM, Miller JW (eds): Albert and Jakobiec's Principles and Practice of Ophthalmology, ed 3. Philadelphia, Saunders Elsevier, 2008, pp 2903-2912.

2 Bullock JD, Fleishman JA, Rosset JS: Lacrimal ductal cysts. Ophthalmology 1986;93:1355-1360.

3 Pfeiffer ML, Yin VT, Bell D, Mancini R, Esmaeli B: Sclerosing polycystic adenosis of the lacrimal gland. Ophthalmology 2013;120:873.

4 Finfer MD, Schinella RA, Rothstein SG, et al: Cystic parotid lesions in patients at risk for the acquired immunodeficiency syndrome. Arch Otolaryngol Head Neck Surg 1988;114:1290-1294.

5 Boulos PR, Harissi-Dagher M, Kavalec C, Hardy I, Codere F: Intralesional injection of Tisseel fibrin glue for resection of lymphangiomas and other thin-walled orbital cysts. 2005;21:171-176. 